This item was submitted to Loughborough's Research Repository by the author.

Items in Figshare are protected by copyright, with all rights reserved, unless otherwise indicated.

\title{
Experimental and numerical analysis of a sedimentation forming compressible compacts
}

PLEASE CITE THE PUBLISHED VERSION

http://www.tandfonline.com/doi/abs/10.1080/01496399708000761

\section{PUBLISHER}

(c) Marcel Dekker inc

\section{VERSION}

AM (Accepted Manuscript)

LICENCE

CC BY-NC-ND 4.0

\section{REPOSITORY RECORD}

Holdich, R.G., and G. Butt. 2012. "Experimental and Numerical Analysis of a Sedimentation Forming Compressible Compacts". figshare. https://hdl.handle.net/2134/9311. 
This item was submitted to Loughborough's Institutional Repository (https://dspace.lboro.ac.uk/) by the author and is made available under the following Creative Commons Licence conditions.

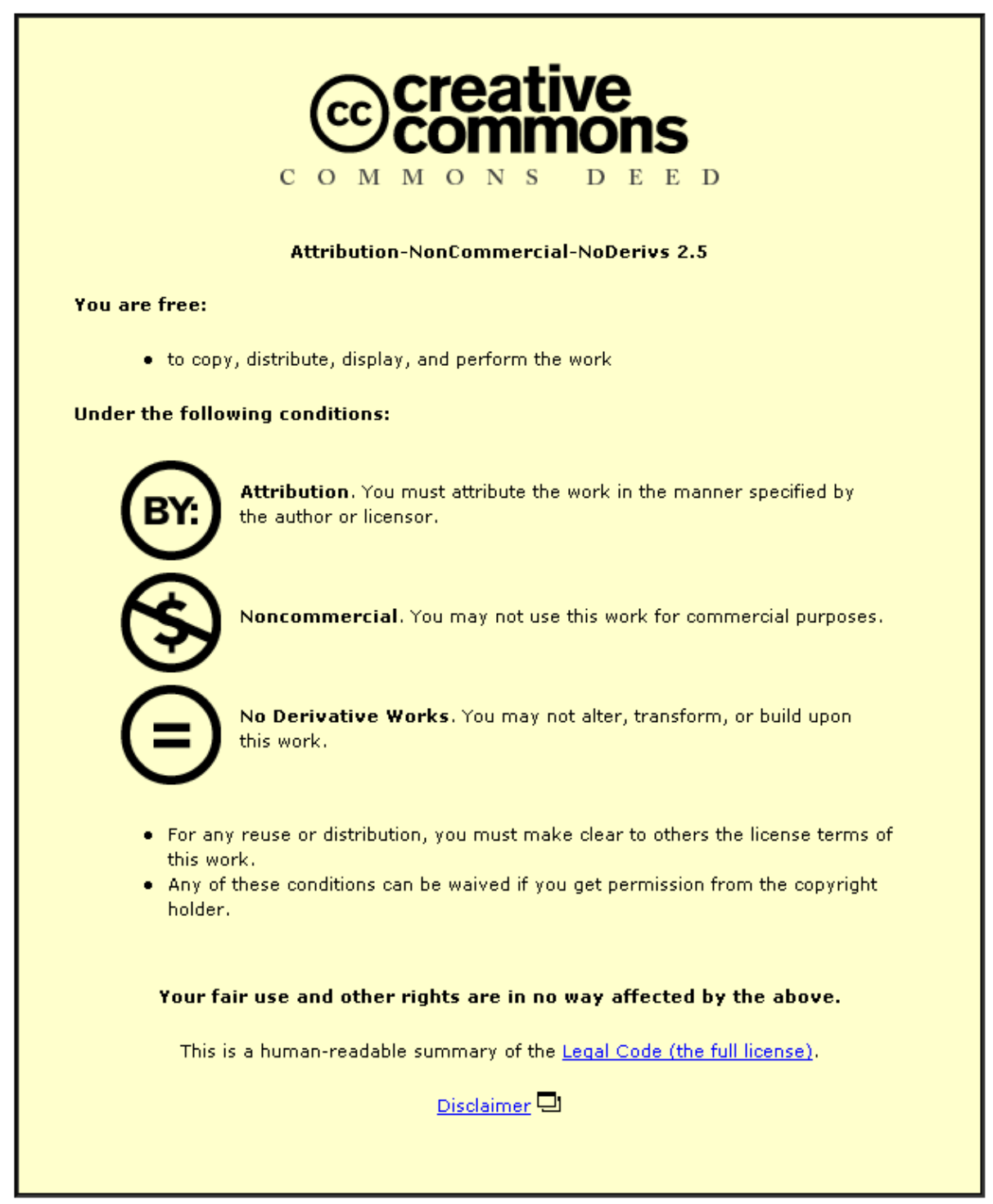

For the full text of this licence, please go to: http://creativecommons.org/licenses/by-nc-nd/2.5/ 


\section{EXPERIMENTAL AND NUMERICAL ANALYSIS OF A SEDIMENTATION FORMING COMPRESSIBLE COMPACTS}

Holdich, R.G and Butt, G. Department of Chemical Engineering, Loughborough University, Leicestershire, UK, LE11 3TU.

Revised paper submitted for publication: 13 January, 1997 


\begin{abstract}
Batch sedimentations of the mineral talc suspended in water at various initial concentrations resulted in compacts that displayed compression, and compression with channel formation. During the experiments the local concentration was deduced by means of local electrical resistance measurement. The technique provided concentrations that integrated throughout the vessel to give masses that matched the known initial mass employed to within $\pm 5 \%$. Two types of channel zones were observed: soft and hard, the former appeared to be due to the liquid inertia of water discharging from the latter. The region within and above the soft channel zone diluted from the initial concentration, and this caused the visible interface between the suspension and the supernatant to accelerate. The top of the hard channel zone followed the line of constant solids concentration representing the first significant increase in concentration over the initial suspension.
\end{abstract}

A finite difference numerical model of sedimentation matched the experimental data, including the data determined below the visible interface, with very high precision for the talc suspensions exhibiting compression with insignificant channelling. The implicit model was implemented on a conventional computer spreadsheet package and rapidly converged. The model did not employ a function for hydraulic permeability, instead a linear function between the socalled Kozeny 'constant' (or coefficient) and concentration was used.

In order to provide an accurate numerical model for compressible sedimentation with significant channel formation the hydraulic permeability needs to be augmented, or the Kozeny coefficient reduced, and the dilution above the channel zone must be predicted. These should be achieved in a way that is general to all sedimentations of a given type of material, and not specific to only one starting concentration.

Experimental and numerical results also indicate that the buoyancy force experienced by the solids is adequately described by the density difference between the solids and the suspending liquid, and not the density difference between the solids and the suspension. 
Gravity sedimentation is a widely practised operation in many industries. Process scale sedimentation equipment is generally designed after performing tests on a small laboratory scale. The laboratory tests identify the appropriate settling regime, and can also generate operating data suitable for use in the design. Laboratory tests may also be employed when investigating the performance of existing process scale sedimentations; identifying possible operating conditions and settling behaviour. The different settling regimes have been described by Fitch (1), and are illustrated in Figure 1.

Clarification, or free settling, is restricted to conditions of low solid concentration and particles may settle as individual units or agglomerates unaffected by the presence of other particles. At solid concentrations greater than 1 or $2 \%$ by weight hindered settling occurs, and particles settle en-masse rather than as individual units. However, when sedimenting in the hindered settling regime further subdivision into zone settling, compression and compression with channelling must be made. In the instance of zone settling alone the particles form incompressible suspensions and sediments where the settling velocity is a unique function of solid concentration, and may be described by well known correlations (2). When undergoing compression the particle settling velocity is a function of solid concentration and other local conditions such as the compressive stress gradient within the sediment.

The mathematical description of sedimentation, leading to design procedures for batch and continuous thickeners, started with the work of Coe and Clevenger (3) and was advanced in the 1950's by Kynch (4), Talmage and Fitch (5) and Yoshioka (6). The designs were based on the assumption of incompressible discrete particles, where settling velocity is a unique function of local concentration. Mathematical techniques for the design of thickeners with due account of solid compact compression were advanced in the 1960, 70 and 80's (7, 8, 9, 10, 11, 12, 13). However, in most instances the occurrence of channels within the compressing sediment was neglected, as a simple means to incorporate channelling into the thickening model was not 
possible. The significance of channels in the overall sedimentation process has been recognised $(1,10,14,15,16,17,18)$, and some thickener geometries have been proposed to encourage this phenomenon (19).

In recent years several research workers have used numerical methods to solve the differential equations for sedimentation of materials giving rise to compressible sediments $(9,20,21,22$, 23). The usual method of assessing the relevance of the numerical model was to compare the numerically predicted interface settling curve, i.e. the height of the interface between the supernatant clear liquid and the settling suspension with respect to time, with the experimentally observed settling curve. This is not a satisfactory test of the applicability of a numerical model as the constituent equations for the model often include terms that have to be determined from such settling tests, and an element of curve fitting may be suggested in some instances.

Obtaining data to investigate the constituent terms is a frequently occurring problem. In the absence of detailed data on local solid concentrations underneath the settling interface the only concentrations and velocities that appear to be reliably known are those of the initial suspension concentrations. However, the occurrence of channels can change these values significantly by dilution of the suspension just below the settling interface $(17,18)$. One of the most difficult to assess constituent equations is the variation of permeability with solid concentration, as the presence of channels will have a considerable influence on results deduced by applying a force balance to data taken from the interface settling plot. The usual approach in this instance is to neglect compression and channelling, and simply to equate the solid weight and liquid drag terms in the force balance, rearrange for hydraulic permeability and substitute in the known initial concentration and measured settling velocity together with other known physical constants. Permeability can be deduced from data taken from the settling curve only when that curve does not display significant compression or dilution; indicated by a curve bending towards or away from the origin, respectively. 
The above restrictions limit the calculation of hydraulic permeability from interface settling data to suspensions of low initial concentration, where channelling and compression are not significant for a substantial part of the settling curve. However, in a numerical model of compressible sedimentation the permeability at moderate and high concentrations is very important for the solution. The approach adopted in this work was to use the settling data from sedimentations at low initial concentrations, in the absence of significant compression and channelling, to use a technique described by Font (14) to estimate permeability at higher concentrations. On comparison of these two methods for permeability analysis with the Kozeny relation, it was discovered that if the Kozeny constant was allowed to vary linearly as a function of concentration the Kozeny permeability relation gave an exact match to the permeability measured by the two experimental methods. The approach of allowing the so-called Kozeny 'constant' to vary with concentration in sedimentation analysis has been suggested before (24). The advantage of using this approach here was that the linear variation of the Kozeny constant, at least over the range of concentrations of interest in this study, made extrapolating the permeability data to high concentration values more reliable than the use of any alternative permeability expression. Also, the application of the Kozeny permeability expression provided, after cancelling, a much simplified coefficient in the partial differential equation describing the sedimentation. This coefficient contained only terms for solid concentration and the Kozeny 'constant', as described later.

The modelling work was also used to investigate the varying contribution from the suspended solids to the liquid pressure gradient surrounding the particle, and hence the buoyancy force. In sedimentation the buoyed weight force per unit area within a force balance conducted on settling particles is

$$
C g\left(\rho_{S}-\rho\right) \mathrm{d} x
$$


where $C$ is the solid concentration in volume fraction, $g$ is the acceleration due to gravity, $x$ represents distance from the base of the column and $\rho_{S}$ and $\rho$ are the solid and liquid densities respectively. A particle in suspension experiences a buoyancy force because of the surrounding liquid pressure gradient and the surrounding liquid pressure gradient is increased due to the presence of particles, thus the buoyed weight term may be written as

$$
C g\left(\rho_{S}-\rho_{m}\right) \mathrm{d} x
$$

where $\rho_{m}$ is the mean density of the suspension including the suspended particles. If the mean suspension density is given by its component densities in appropriate volume ratios the buoyed weight term becomes after simplification

$$
C g\left(\rho_{S}-\rho\right)(1-C) \mathrm{d} x
$$

It has been argued $(8,25)$ that when the particles are fully suspended the above equation is valid, but when the particles are fully settled the equation must revert to that given initially. Hence the term $(1-C)$ is the buoyancy correction term varying from a fractional value to unity during sedimentation. For the sedimentation of largely incompressible materials the change from a fractional value to unity is sudden; at the end of the settlement period. However, for the sedimentation of compressible compacts a network of solids that can transmit the suspended particle weight to the base of the vessel will slowly build up, thus the buoyancy correction term will slowly vary from $(1-C)$ at the start of the sedimentation to unity at the end. This makes the buoyancy correction term a function of the sedimentation history, and the numerical modelling of the process will also become complicated. The numerical and experimental investigations of talc sedimentations also considered the significance, or otherwise, of the change in the buoyancy correction term. 


\section{MODEL DEVELOPMENT}

A force balance on a settling layer of suspension during batch sedimentation in Cartesian coordinates gives

$$
\left.\frac{\partial P_{S}}{\partial x}\right|_{t}=C g\left(\rho_{S}-\rho\right)-\frac{\mu}{k} v
$$

where $\partial P_{S} / \partial x$ is the solids compressive stress gradient $k$ is the hydraulic permeability, $v$ is the solid velocity and $\mu$ is the liquid viscosity. Defining the mass of solids per unit area to be ' $w$ ' then

$$
w=C \rho_{s} \delta x
$$

The total mass of solids up to a height $x$ is

$$
w_{0}=\int_{0}^{x} C \rho_{s} \mathrm{~d} x
$$

Equation (2) can be used to transfer the system to material co-ordinates. In this system the total mass of solids in a batch sedimentation vessel is split into a number of elements, each containing the same mass of material. Only at the start of the sedimentation are the elements of equal volume, i.e. area and height. The material co-ordinate system is illustrated schematically in Figure 2.

Darcy's law, written in a material co-ordinate system and combined with a liquid force balance gives

$$
v=\frac{k C \rho_{S}}{\mu} \frac{\partial P_{L}}{\partial w}
$$

where $P_{L}$ is the liquid pressure in excess of the hydrostatic pressure due to the presence of suspended solid material. The continuity equation in material coordinates is

$$
\frac{1}{\rho_{s}} \frac{\partial C}{\partial t} \frac{1}{C^{2}}=-\frac{\partial v}{\partial w}
$$


Combining Equations (1) to (4), employing the Chain Rule and rearranging provides the following equation for the variation in the excess liquid pressure, as a function of time, due to the settlement of a compressible compact

$$
\frac{\partial P_{L}}{\partial t}=-\frac{\rho_{S} C^{2}}{\mathrm{~d} C / \mathrm{d} P_{S}} \frac{\partial}{\partial w}\left[\frac{k C \rho_{S}}{\mu} \frac{\partial P_{L}}{\partial w}\right]
$$

which is a parabolic equation in excess liquid pressure. A suitable choice of coefficients can be made to make Equation (5) non-dimensional, these are

$$
\begin{gathered}
w_{0}=H_{0} C_{0} \rho_{s} \\
P_{0}=w_{0}\left(\rho_{s}-\rho\right) g / \rho_{s} \\
P_{L}^{*}=P_{L}\left[\frac{\rho_{s}}{g\left(\rho_{s}-\rho\right) w_{0}}\right] \\
P_{S}^{*}=\frac{P_{S}}{P_{0}} \\
w^{*}=\frac{w}{w_{0}} \\
t^{*}=\frac{t}{t_{0}}
\end{gathered}
$$

where the non-dimensional terms have asterisks, and the non-dimensionalising time function $t_{0}$ is defined as follows

$$
t_{0}=\frac{H_{0}}{v_{0}}
$$

The non-dimensionalising function of solids settling velocity $\left(v_{0}\right)$ is defined as follows

$$
v_{0}=\frac{\left(1-C_{0}\right)^{3}}{K_{0} C_{0} S^{2}} \frac{g\left(\rho_{s}-\rho\right)}{\mu}
$$

where $S$ is the specific surface area per unit volume of the solid particles and $K_{0}$ is the Kozeny constant in the Kozeny-Carman permeability expression, and typically has the value of 5 for static beds and 3.36 for moving beds. It is often found that the Kozeny permeability expression 
provides a good representation of the functional relation between hydraulic permeability and solid concentration provided an empirical value is used for the Kozeny constant (24). Furthermore, the Kozeny constant is a term that includes a tortuousity factor and various other numbers that have their origins in the transformation of the Hagen Poiseuille equation into the Kozeny-Carman equation. There is no reason why these factors and numbers should be constant with variable concentration. Hence it is a reasonable approach to allow the Kozeny 'constant' to become a variable dependent on solid concentration (24). Under these circumstances it is more meaningful to use the phrase Kozeny 'coefficient' rather than constant. The correct Kozeny coefficient to use in Equation (13) is the value appropriate to the concentration $C_{0}$. Equation (13) physically represents the hindered settling velocity when there is no stress gradient $\left(\partial P_{S} / \partial x=0\right)$, and can be derived from Equation (1) by using the Kozeny permeability expression.

After making the appropriate substitutions Equation (5) becomes

$$
\left(\frac{\mathrm{d} C}{\mathrm{~d} P_{S} *} \frac{1}{C^{2}}\right) \frac{\partial P_{L}^{*}}{\partial t^{*}}=-\frac{\partial}{\partial w}\left[\frac{\rho_{S} P_{o} t_{o} k C \rho_{S}}{w_{o}{ }^{2} \mu} \frac{\partial P_{L}^{*}}{\partial w^{*}}\right]
$$

where the subscript 'o' refers to non-dimensionalising functions for pressure, time and mass of solids per unit area. Equation (14) is a parabolic equation with non-linear coefficients and is of the form

$$
b\left(P_{L}^{*}\right) \frac{\partial P_{L}^{*}}{\partial t^{*}}=\frac{\partial}{\partial w}\left[a\left(P_{L}^{*}\right) \frac{\partial P_{L}^{*}}{\partial w^{*}}\right]
$$

and can be solved numerically, under appropriate boundary and initial conditions, by a finite difference method (26). The function $b\left(P_{L}{ }^{*}\right)$ can be assessed once the functional relation between solid compressive pressure and concentration has been determined, and in combination with Equations (6), (7) and (9). The experimental equipment provided a means to measure the 
final settled sediment concentration profiles, thus these profiles were used in the analysis of solid concentration as a function of compressive pressure.

When the sedimentation is complete Equation (1) is simplified as the settling velocity is zero, and the local solids pressure can be obtained by integrating the concentration profile between the height under investigation and the surface of the sediment. Thus the total solid pressure within the vessel is

$$
P_{s}=\left(\rho_{s}-\rho\right) g \int_{0}^{\mathrm{H}} C \mathrm{~d} x
$$

where $H$ is the sediment height. The functional relation between concentration and solid pressure was described adequately by the following equation

$$
C=C^{\prime} P_{s}^{n}
$$

where $C^{\prime}$ and $n$ are empirical constants and had the values of 0.088 and 0.144 , respectively, for the talc mineral employed in this study.

Using Equations (6), (7), (9) and (15) gives the following equation for the function $b\left(P_{L}^{*}\right)$ after suitable simplification

$$
b\left(P_{L}^{*}\right)=\frac{n H_{0} C_{0}\left(\rho_{s}-\rho\right) g}{C \rho_{s}}
$$

The function $a\left(P_{L}{ }^{*}\right)$ contains the hydraulic permeability which is a function of local solid concentration. However, if the Kozeny permeability expression is used, i.e.

$$
k=\frac{(1-C)^{3}}{K C^{2} S^{2}}
$$

where $K$ is the Kozeny coefficient, and Equations (12) and (13) are substituted into the function then the permeability term cancels from the expression together with the specific surface area per unit volume of the solids. The resulting expression for the function $a\left(P_{L}{ }^{*}\right)$ is 


$$
a\left(P_{L}^{*}\right)=\frac{K_{0}}{\left(1-C_{0}\right)^{3}}\left[\frac{(1-C)^{3}}{K C}\right]
$$

where $K_{0}$ is Kozeny coefficient at the concentration $C_{0}$. The term within the square bracket on the right side of Equation (16) contains the local concentration and the Kozeny coefficient, which is also dependent on the local concentration. If the Kozeny coefficient was, in fact, a constant Equation (16) would simplify considerably. Such an approach may be valid as a first approximation in a sedimentation analysis, but in this study Equation (16) was used after tests to evaluate the concentration dependency of the permeability and, thereby, the Kozeny coefficient. The functional relation between permeability and solid concentration was deduced following the technique described by Font (14), applied to one set of the experimental results. The analysis requires the local solids settling velocity calculated from a modified material balance

$$
v=\frac{1}{C}\left[C_{*} \nu_{*}-\frac{\mathrm{d} L}{\mathrm{~d} t}\left(C-C_{*}\right)+\int_{0}^{\mathrm{x}} \frac{\partial C}{\partial t} \mathrm{~d} x\right]
$$

where $C_{*}$ is the concentration at the top of the sediment (in this analysis the concentration of the first line of constant concentration from the base of the vessel), $v_{*}$ is the settling velocity of that concentration when it reaches the settling interface and $\mathrm{d} L / \mathrm{d} t$ is the rate of propagation, upwards from the base, of the sediment concentration at the time $t$. A knowledge of the local concentration with time is needed in order to apply Equation (17). According to Font's technique the local velocity obtained from Equation (17) can then be used in Equation (18) to provide a value for permeability

$$
k=\frac{\mu v}{C g\left(\rho_{S}-\rho\right)-\left.\left(\frac{\mathrm{d} P_{S}}{\mathrm{~d} C}\right)\left(\frac{\partial C}{\partial x}\right)\right|_{t}}
$$

Again a knowledge of the local concentration, this time with respect to distance within the column, is required in order to apply Equation (18) and the functional relation between solid 
pressure and concentration must be known. The original reference contained a typographical error in the reproduction of this equation: Equation (30) of reference (14), but Equation (18) above is the correct form of this expression.

Given the appropriate constituent equations, an explicit finite difference solution to Equation (14) was attempted but found to be unstable. Thus an implicit three time level method (26) was employed as follows

$$
\begin{gathered}
P_{L}{ }^{*}{ }_{i+1, j}=\frac{1}{3}\left(P_{L}{ }^{*}{ }_{i+1, j+1}+P_{L}{ }^{*}{ }_{i+1, j}+P_{L}{ }^{*}{ }_{i+1, j-1}\right) \\
P_{L}{ }^{*} i, j \\
=\frac{1}{3}\left(P_{L}{ }^{*}{ }_{i, j+1}+P_{L}{ }^{*} i, j+P_{L}{ }^{*}{ }_{i, j-1}\right) \\
P_{L}{ }^{*}{ }_{i-1, j}=\frac{1}{3}\left(P_{L}{ }^{*}{ }_{i-1, j+1}+P_{L}{ }^{*}{ }_{i-1, j}+P_{L}{ }^{*}{ }_{i-1, j-1}\right)
\end{gathered}
$$

where $i$ and $j$ represent dimensionless material and time co-ordinates. The finite difference solution to Equation (14) using this approach is

$$
\begin{aligned}
& P_{L}^{*}{ }_{i, j+1}=\left[\frac{3 b\left(C_{i, j}\right)}{2 \Delta t^{*} / \Delta w^{* 2}} P_{L}{ }^{*}{ }_{i, j-1}+a\left(\frac{1}{2}\left[C_{i+1, j} ; C_{i, j}\right]\right)\left(P_{L}{ }^{*}{ }_{i+1, j+1}+P_{L}{ }^{*}{ }_{i+1, j}+P_{L}{ }^{*}{ }_{i+1, j-1} \ldots\right.\right. \\
& \left.\ldots-P_{L}{ }^{*} i, j-P_{L}{ }^{*} i, j-1\right)-a\left(\frac{1}{2}\left[C_{i-1, j} ; C_{i, j}\right]\right)\left(P_{L}{ }^{*} i, j+P_{L}{ }^{*} i, j-1-P_{L}{ }^{*}{ }_{i-1, j+1}-P_{L}{ }^{*} i+1, j \ldots\right. \\
& \left.\left.\ldots-P_{L}{ }^{*}{ }_{i-1, j-1}\right)-\right]\left[\frac{3 b\left(C_{i, j}\right)}{2 \Delta t^{*} / \Delta w^{* 2}}+a\left(\frac{1}{2}\left[C_{i+1, j} ; C_{i, j}\right]\right)+a\left(\frac{1}{2}\left[C_{i-1, j} ; C_{i, j}\right]\right)\right]^{-1}
\end{aligned}
$$

The initial condition used in the solution was

$$
P_{L}^{*}=1-w^{*-} P_{S o}^{*}
$$

where $P_{S o}{ }^{*}$ is the non-dimensionalised form of the initial solid stress. The boundary conditions were

$$
\frac{\partial P_{L}^{*}}{\partial w^{*}}=0 \quad \text { at } w^{*}=0
$$




$$
P_{L}^{*}=0 \quad \text { at } w^{*}=1
$$

The use of a three time level method necessitated a pseudo initial row before the row at $j=0$. This pseudo initial row was assumed to have the same values as the true initial condition row. The model was solved iteratively on a standard computer spreadsheet package using the cells within the spreadsheet pages to represent the elemental cells for the numerical model. The solution used 6 spreadsheet pages for values of:

$$
P_{L}^{*}{ }_{i, j} \quad ; C_{i, j} \quad ; a\left(\frac{1}{2}\left[C_{i+1, j} C_{i, j}\right]\right) ; a\left(\frac{1}{2}\left[C_{i-1, j} C_{i, j}\right]\right) ; P_{s i, j} ; b\left(C_{i, j}\right)
$$

Each spreadsheet cell location on each page represented the value of the appropriate function under the same conditions of $i$ and $j$. The values used in the solution for $\Delta w^{*}$ and $\Delta t^{*}$ were 0.05 and 0.00125 respectively. The spreadsheet function of iterative calculation of the cell values was employed until convergence of the cell values was observed. The model ran on a Pentium IBM compatible PC at $90 \mathrm{MHz}$, with 16MB of RAM, and was generally completed within 5 minutes. A further page was used for the calculation of the height from the base corresponding to each value of $i$ and $j$. The total height of the suspension undergoing compression was calculated from

$$
h=\sum_{i=1}^{25} \frac{\Delta w_{i}}{\rho_{s}\left(C_{i}+C_{i-1}\right) / 2}
$$

\section{EXPERIMENTAL TECHNIQUES}

Two batch settling vessels were used with internal diameters of 61.5 and $140 \mathrm{~mm}$. Both sedimentation vessels were $400 \mathrm{~mm}$ in height, and the initial suspension height was $331 \mathrm{~mm}$ in all of the experiments. The equipment was housed in an insulated wooden box to provide a constant temperature environment. Temperature control to $25 \pm 0.5{ }^{\circ} \mathrm{C}$ was maintained by a 
thermostat connected to two Radio Spares heating mats, or two $240 \mathrm{~V}$ light bulbs for the smaller rig. In all the tests the local concentration was measured by using vessels equipped with electrodes to record the local electrical resistance and hence concentration, see Figure 3. Full details of the experimental equipment have been described before (27).

The calibration of electrical conductivity ratio (ratio of the continuous phase and mixture phase conductivity) to solid concentration by volume fraction requires the knowledge of one empirical constant $(m)$

$$
C=1-\sqrt[m]{\frac{\kappa_{m}}{\kappa_{c}}}
$$

where $\kappa_{m}$ and $\kappa_{c}$ are the mixture and continuous phase conductivities respectively. The experimental equipment provided values of the local conductivity ratio throughout the sedimentation. The empirical constant that provided the lowest error in the mass balance on the talc solids for all the sedimentations and at all sedimentation times was used to convert the conductivity ratios to concentrations. The mass balance compared the known original mass of solids added to the vessel with that obtained by integrating the concentration profile at all settling times

$$
A \rho_{s} \int_{0}^{H} C d x
$$

where $A$ is the area of the sedimentation vessel and $H$ is the height of the suspension. The percentage error in the mass balance using an exponent value of 1.98 ranged between $\pm 5 \%$ at the extremes, but was generally within the range $\pm 2 \%$.

The variation in the liquid pressure gradient due to solids settlement was measured by placing two very sensitive pressure transducers $204 \mathrm{~mm}$ apart in the larger diameter sedimentation vessel. The transducers were Druck type PDCR 810, with a full scale reading of 70 mbarg corresponding to a $17 \mathrm{mV}$ output. The output from the transducers were measured using two digital volt meters (DVM) with 4.5 digit resolution (Black Star model 4310). The outputs from 
the transducers measured by the DVMs were calibrated against a column of water of various heights. Other experimental conditions were similar to those described, except that the initial concentration was very high at $17.5 \%$ by volume. The use of such a high starting concentration ensured that channelling was not significant during the sedimentation. The final sediment concentration was at a reasonably uniform value of $22.5 \%$. During the sedimentation, and after settlement, the suspension height was always well above the top transducer. The settlement of solids reduced the readings from both transducers. There was also a reduction in pressure difference between these two transducers which was due to the solid weight becoming transmitted to the base of the vessel by a developing solid network. This experiment was designed to test the theory that buoyancy correction may be a complicated function of sedimentation history, as described in the Introduction.

In all of the experiments the liquid phase was a weak $\left(10^{-5} \mathrm{M}\right)$ potassium nitrate solution. This very dilute electrolyte was employed as a buffer to provide a constant ionic strength against any slight dissolution of ionic material from the surface of the talc mineral. It also enhanced the conductivity of the distilled water making up the continuous phase and thus facilitated the use of electrical conductivity measurement. The mineral density was determined by using density bottles and distilled water, and the size distribution of the solids was obtained on a Coulter Electronics LS 130, a device that uses both Fraunhofer laser light diffraction and light diffusion analyses. The particle shape was discovered to be a platelet type, similar to that of a clay mineral, from images obtained by a scanning electron microscope. The physical properties of the talc mineral employed in this study are given in Table 1.

\section{DISCUSSION OF EXPERIMENTAL RESULTS}


Channels were observed during the sedimentations, the most significant channelling occurred when settling suspensions with initial concentrations between 7 and $15 \%$ by volume. In all instances it was possible to deduce two distinct channel zones: hard and soft. These zones are illustrated in Figure 4, the lower hard zone had distinct channel walls that stayed in the same location for a significant length of time. The suspension phase surrounding the hard channels appeared to have a more cohesive structure than the suspension surrounding the soft channels. The channels within the soft zone were smaller and prone to move, whilst maintaining a base positioned directly above a hard channel. The general appearance of the suspension surrounding the soft channels was that of a fluidised system. Thus it may be concluded that the soft channels are due to liquid inertia caused by discharge from the hard channels. In all these respects the talc mineral behaved in a similar way to the sedimentation of calcite described elsewhere (18).

Figure 5 shows the interface height with time, together with the iso-concentration curves or 'lines of constant solids concentration' formed during the batch sedimentation of a suspension at an initial uniform concentration of $7.2 \%$. The coincidence between the top of the hard channel zone and the line of constant solids concentration representing the first significant increase in concentration over the feed is evident. This observation was found to be true for all the initial suspension concentrations investigated. The electrical conductivity scanning equipment indicated that the concentration above the channel zone but still below the settling interface diluted to values below the starting concentration. Thus during sedimentation the interface velocity increased because of 3 factors: a lowering of the solid concentration below the interface, on breakthrough of soft channels to the surface, and on breakthrough of hard channels to the surface. On the occurrence of these last two phenomena the interface curve accelerates very rapidly. The settled sediment concentration profiles for all the tests were used to provide values for the constants in Equation (15), following the procedure in the section on Model Development detailed just prior to Equation (15). This resulted in values of 0.088 and 0.144 for the coefficient 
and exponent, respectively. Consideration of these values leads to the conclusion that compressive stress or solid pressure is insignificant at concentrations below $4 \%$ by volume, and this value represents the limit of applicability of Equation (15). Observations made of the settling behaviour (eg the concentration profiles) of suspensions just above and below this value also suggested that the critical solids concentration, marking the boundary between the hindered settling zone and the compression zone, is close to a concentration of $4 \%$ by volume.

Hydraulic permeability was estimated from the initial settling rates of suspensions at concentrations of 2 and $5.2 \%$ by volume, by equating the solid weight and liquid drag terms in the force balance, rearranging for hydraulic permeability and substituting in the known initial concentrations and measured settling velocities together with other physical constants. This was not attempted for higher concentrations as channelling and compression became increasingly significant. The permeability resulting from the analysis using an initial concentration of $5.2 \%$ by volume is likely to be slightly in error, due to the presence of small compressive forces at this concentration. However, permeability was also determined by the method of Font (14), and the resulting values were consistent with those given by the earlier method. Font's technique was applied to the experimental data obtained from the sedimentation at an initial concentration of $7.2 \%$ by volume, and the procedure given in Equations (17) and (18) was used to provide permeability values up to a concentration of $18 \%$ by volume. For the purpose of numerical modelling permeability values of up to $22 \%$ by volume were required, hence the Kozeny permeability expression was compared to the deduced permeability values. When using a linearly varying Kozeny coefficient the Kozeny permeability expression matched the values provided by the two experimental techniques. The variation in the Kozeny coefficient with solid concentration is illustrated in Figure 6. The consistency of the data obtained from the initial settling rates and the technique of Font can be seen from this figure. The linear function between the Kozeny coefficient and concentration was deduced from Figure 6 and used in the 
extrapolation of the Kozeny coefficient up to solid concentrations of $22 \%$ for use in the numerical model.

Equation (20) was solved, using the spreadsheet finite difference model, for an initial solid concentration of $5.2 \%$ by volume. The result of the solution is compared to the experimental measurements in Figure 7. At this concentration only limited channelling was observed, and the very close agreement between the model and measured values indicate that compression was significant for all the concentrations present during sedimentation, but that the channelling can be ignored. Figure 8 illustrates the concentration profile within the batch sedimentation vessel after settling for 22,000 seconds. Again the agreement between the experimentally measured values of concentration with height and those provided by the numerical model is excellent. The comparison between the model and experimental data illustrated in Figures 7 and 8 are regarded as a more rigorous test of a numerical compression model than is given by simply comparing the interface settling height with respect to time; both the interface and local concentrations underneath the interface are correctly positioned. Thus it may be concluded that the numerical model accurately represents the compression of the talc mineral in the absence of significant channelling effects. It is worth noting that the permeability data used to provide one of the constituent equations used in the model was derived empirically, but from a test using a different intial concentration to the test numerically modelled.

Figure 9, however, illustrates the significance of channelling on the compressible talc system. In this instance the initial suspension concentration was $11.2 \%$ by volume, and significant channelling was observed. The initial rate of descent of the settling interface, and the final sediment height, are correctly predicted by the numerical model considering compression only, but there is considerable discrepancy between all the predicted and measured lines of constant solids concentration and with the interface settling curve after 14,000 seconds. The occurrence of channels appears to increase the speed with which all lines of constant solids concentration 
propagate from the base of the vessel, even those that are at concentrations in excess of those supporting channels. The bottom of the channel zone was not concurrent with a line of constant solids concentration, unlike the top of the zone, the $20 \%$ line of constant solids concentration was always below the channel zone; but the existence of channels is clearly affecting the propagation of this and higher concentrations.

The uniform increase in lines of constant solids concentration position during channelling sedimentation supports the concept of augmenting the permeability, or decreasing the Kozeny coefficient, during the channelling part of the sedimentation by a time dependent function only (13). However, such a time dependent function will only be valid for the sedimentation under consideration, and not applicable to ones starting at a different concentration, etc. Thus augmenting permeability in a numerical model is a useful approach to account for the existence of channels, but the augmentation must be based on a more fundamental basis than a time function. Such a basis does not yet exist and may provide a fruitful area of research.

The experimental results illustrated in both Figures 5 and 9 show some interesting results worthy of further mention: the divergent lines of constant solids concentration in the compression zone. These are evident in Figure 5 from a time of 30,000 seconds (note the 0.17 to 0.19 iso-concentration curves), and to a lesser extent in Figure 9 at concentrations between 0.20 and 0.22 . Curves of such shape have been reported before $(18,28)$ with other mineral suspensions. The shape of these curves cannot be explained by compression alone: they appear to accelerate into the column and are associated with sedimentation displaying channelling.

The last objective of this work was to investigate the varying contribution from the suspended solids to the liquid pressure gradient, and hence the buoyancy force experienced by a particle during sedimentation of a compressible compact. The origin of a variable buoyancy force was described in the Introduction. The numerical model was formulated to calculate the liquid pressure in excess of the hydrostatic at any height within the vessel. The calculation was based 
on the assumption that the buoyancy term is given by the expression $\left(\rho_{s}-\rho\right)$ and not by the term $\left(\rho_{s}-\rho_{m}\right)$. Thus if the liquid pressure in excess of the hydrostatic (excess pore pressure) should be calculated using the latter rather than the former approach then the experimentally measured pressures will be significantly different from those predicted by the numerical model. For this part of the work an initial solid concentration that displayed insignificant channelling but significant compression was required. Adequate mixing of the initial suspension imposed the upper limit of solid concentration usable at $17.2 \%$. The suspension subsided slowly to provide a sediment with an average concentration between the two sensitive pressure transducers of $22.5 \%$. Before sedimentation the excess pore pressure between these two transducers corresponded to approximately $600 \mathrm{~Pa}$, and is in accordance with the equation

$$
\Delta P=\Delta h\left(\rho_{m}-\rho\right) g
$$

where $\Delta h$ is the height difference between the transducers and

$$
\rho_{m}=C \rho_{s}+(1-C) \rho
$$

These two equations are only applicable prior to compression taking place.

During sedimentation the excess pore pressure reduced to zero; i.e. liquid pressure reduced to that of the hydrostatic head alone, as solids settled out of suspension and all the particle weight became supported by a solid network from the base of the vessel. The numerical model predicts this behaviour assuming that the buoyancy term remains $\left(\rho_{s}-\rho\right)$ throughout the process, and is not given by the term $\left(\rho_{s}-\rho_{m}\right)$, or the term $\left(\rho_{s}-\rho_{m}\right)$ together with a time dependent function taking a value between $(1-C)$ and unity as explained earlier. The results of this experiment and the numerical simulation, up to a time of 75,000 seconds, are given in Figure 10.

The excellent agreement between the predicted and measured excess pore pressure indicates that the numerical model was correctly formulated, and concerns over the use of any function for buoyancy correction other than $\left(\rho_{s}-\rho\right)$ appears unfounded, at least in systems similar to that 
described here. This is a reassuring result as a varying buoyancy correction term would otherwise need to be incorporated into the numerical model, and it is not immediately apparent what sort of function the correction term would take.

\section{CONCLUSIONS}

Numerical models of sedimentation of compressible compacts have been available for some time. However, most of the experimental work reported with these models have considered only the rate of fall of the settling interface, and used these measurements to obtain data for application in the model. Often interface settling curves, bending away from the origin, were observed which are an indication of significant dilution below that interface and, therefore, will reduce the reliability of data deduced from the settling interface. In the study reported here an experimental technique provided a comprehensive set of results on the local conditions below the settling interface. Hydraulic permeability at low concentration was deduced by two experimental techniques, and the method of Font (14) was discovered to be reliable for low and intermediate concentrations. A variable Kozeny coefficient, which displayed a linear function with solid concentration was apparent, which was used to extrapolate to high concentrations. The linear Kozeny coefficient function was incorporated into the numerical model, resulting in an expression that was dependent only upon concentration and the Kozeny coefficient, and not explicitly on permeability.

For stable numerical solutions the finite difference numerical model required an implicit (iterative) solution technique, and was implemented on a conventional computer spreadsheet. Convergence occurred in only a few minutes. The model successfully predicted the sedimentation behaviour of talc suspensions at initial concentrations below those at which significant channelling was observed. However, in cases of significant channelling the experimentally measured lines of constant solids concentration were positioned considerably 
higher in the vessel than the predicted ones, even at concentrations below the channel zone. Hence channelling increases the speed with which all concentrations form and propagate during sedimentation. Augmentation of the hydraulic permeability, or reduction of the Kozeny coefficient, may be one method to account for channelling within the numerical model, but this could only be achieved by 'curve-fitting' the predicted and measured lines of constant solids concentration. The function chosen to augment the permeability should be of general applicability to sedimentations at different starting concentrations, rather than a time dependent function that will be applicable to sedimentation at only one initial concentration. Such an approach does not yet exist.

A further complication to the numerical modelling of sedimentation with channelling is the dilution that occurs below the settling interface. Dilution below the interface is one of the main causes of the curvature away from the origin that the interface displays, and an accurate numerical model should incorporate this phenomenon. At the bottom of the region in which dilution occurred 'soft' channels exist. These appeared to be channels that owed their existence to the discharge of liquid from the underlying 'hard' channels. The suspension surrounding the soft channels was in a semi-fluidised, and well mixed, state. This condition will assist the uniform dilution of the suspension below the interface.

Liquid pressure measurements compared with the model prediction showed that the buoyancy correction within the weight term of the force balance is correctly given by the expression $\left(\rho_{s}-\rho\right)$ and not by the term $\left(\rho_{s}-\rho_{m}\right)$. Thus there does not appear to be a functional relation between buoyancy force and compressive pressure during sedimentation, at least for the material and conditions reported here.

\section{REFERENCES}


1. $\quad$ Fitch, E.B., "Sedimentation process fundamentals", Trans. Am. Inst. Min. Eng., 223, 129-137 (1962).

2. Garside, J. and Al-Dibouni, M.R., "Velocity-voidage relations for fluidisation and sedimentation in solidliquid systems", Ind. Eng. Chem. Proc. Des. Dev., 16, 206 (1977).

3. Coe, H.S. and Clevenger, G.H., "Methods for determining the capacities of slimes settling tanks", Trans. Am. Inst. Min. Met. Eng, 55, 356-384 (1916).

4. Kynch, G.J., "A theory of sedimentation", Trans. Faraday Society, 48, 166-177 (1952).

5. Talmage, W.P. and Fitch, E.B., "Determining thickener unit areas", Ind. Eng. Chem., 47, 38-41 (1955).

6. Yoshioka, N., Hotta, Y. and Tanaka, S., "Continuous thickening of homogeneous flocculated slurries", Soc. Chem. Eng. Japan, 2, 47-54 (1957)

7. Michaels, A.S. and Bolger, J.C., "Settling rates and sediment volumes of flocculated kaolin suspensions", Ind. Eng. Chem. Fund., 1, 24-33 (1962).

8. Schiffman, R.L., Pane, V. and Sunara, V., "Sedimentation and consolidation", Engineering Foundation Conference, Sea Island, 1985, AIChE, New York, 57-121.

9. Hoyland, G., "A new design procedure for consolidation tanks", in Effluent Treatment and Disposal, IChemE Symp. Ser. No. 96, 119-136 (1986).

10. Kos, P., "Gravity thickening of sludges", PhD Dissertation, University of Massachusetts, Amherst, MA USA (1978).

11. Dixon, D.C., in Progress in Filtration and Separation, Vol 1, ed R.J. Wakeman, Elsevier, Amsterdam, 1979.

12. Tiller, F.M., "Revision of Kynch sedimentation", AIChE J., 27, 823-829 (1981).

13. Font, R., "Analysis of the batch sedimentation test", Chem. Eng. Sci., 46, 2473-2482 (1991).

14. Font, R., "Permeability values from batch tests of sedimentation", Ind. Eng. Chem. Res., 33, 2859-2867 (1994).

15. Dell, C.C. and Kaynar, M.B., "Channelling in flocculated suspensions", Filtration and Separation, 5, 323-327 (1968).

16. Tory, E.M., "Batch and continuous thickening of slurries", PhD Dissertation, Purdue University, USA (1961).

17. Vesilind, P.A. and Jones, G.N., "A reexamination of the batch thickening curve", Research Journal WPCF, 62, 887-893 (1990). 
18. Holdich, R.G. and Butt, G., "An experimental study of channelling and solid concentration during the batch sedimentation of calcite suspensions", Trans. IChemE., 73, Part A, 833-841 (1995).

19. Chandler, J.L., "Dewatering by deep thickeners without rakes", Filtration and Separation, 20, 104-106 (1983).

20. Shirato, M., Kato, H., Kobayashi, K. and Sakazaki, H., "Analysis of settling of thick slurries due to consolidation", J. Chem. Eng. Japan, 3(1), 98-104 (1970).

21. Fujisaki, K. and Amir, S., "Sedimentation and compression of flocculent suspension", 6th World Filtration Congress, Nagoya, Japan, 1993, 100-103.

22. Buscal, R. and White, L.R., "The consolidation of concentrated suspensions", J. Chem. Soc. Faraday Trans., 83, 873-891 (1987).

23. Landman, K.A. and White, L.R., "Solid/liquid separation of flocculated suspensions", Advances in Colloid and Interface Science, 51, 175-246 (1994).

24. Davis, L. and Dollimore, L., "Theoretical and experimental values for the parameter k of the KozenyCarman equation, as applied to sedimenting suspensions", J. Phys. D: Appl. Phys., 13, 2013 (1980).

25. Holdich, R.G., "Buoyancy force in sedimenting and filtering systems", Chem. Eng. Sci., 47, 3167-3168 (1992).

26. Smith, G.D., Numerical Solution of Partial Difference Equations: Finite Difference Methods, Clarendon Press, Oxford, UK, 1979.

27. Holdich, R.G. and Sinclair, I., "Measurement of slurry solids content by electrical conductivity", Powder Technology, 72, 77-87 (1992).

28. Gaudin, A.M. and Fuerstenau, M.C., "Experimental and Mathematical Model of Thickening", Trans. AIME, 226, 122-129 (1962). 
$\underline{\text { Table } 1 \quad \text { Density and particle diameters of talc used in tests }}$

\begin{tabular}{|l|l|l|l|l|l|l|l|l|}
\hline & Density & \multicolumn{6}{|l|}{ Diameters $(\mu \mathrm{m})$ at following mass undersizes(\%): } \\
\hline & $\left(\mathrm{kg} \mathrm{m}^{-3}\right)$ & 100 & 90 & 70 & 50 & 30 & 10 & 0 \\
\hline Talc & 2690 & 40 & 17 & 11 & 7.4 & 4.6 & 2.0 & 0.1 \\
\hline
\end{tabular}




\section{LIST OF FIGURES}

$\underline{\text { Figure } 1}$ Types of sedimentation behaviour according to Fitch (1)

$\underline{\text { Figure } 2} \quad \underline{\text { Schematic illustration of material coordinate system }}$

$\underline{\text { Figure } 3} \quad \underline{\text { Schematic diagram of sedimentation equipment }}$

$\underline{\text { Figure } 4} \quad \underline{\text { Illustration of hard and soft channel zone regions }}$

Figure $5 \quad$ Measured lines of constant solids concentration and observed interface and channel heights from the base with time for a sedimentation at an initial concentration of $7.2 \% \mathrm{v} / \mathrm{v}$

Figure 6 Variation in Kozeny coefficient for talc with solid concentration obtained from permeability data using two different experimental techniques

Figure $7 \quad$ Measured and predicted lines of constant solids concentration for a talc sedimentation at an initial concentration of $5.2 \% \mathrm{v} / \mathrm{v}$

$\underline{\text { Figure } 8} \quad$ Measured and predicted talc concentration profile during a talc sedimentation at an initial concentration of $5.2 \% \mathrm{v} / \mathrm{v}$

Figure $9 \quad$ Measured and predicted lines of constant solids concentration for a talc sedimentation at an initial concentration of $11.2 \% \mathrm{v} / \mathrm{v}$

$\underline{\text { Figure } 10} \quad \underline{\text { Hydrostatic pressures and interface height for a talc sedimentation at an }}$ initial concentration of $17.2 \% \mathrm{v} / \mathrm{v}$ 
Figure 1 Types of sedimentation behaviour according to Fitch (1)

EXPERIMENTAL AND NUMERICAL ANALYSIS OF A SEDIMENTATION FORMING COMPRESSIBLE COMPACTS

R.G. Holdich \& G. Butt 
$\underline{\text { Figure } 2} \underline{\text { Schematic illustration of material coordinate system }}$

EXPERIMENTAL AND NUMERICAL ANALYSIS OF A SEDIMENTATION FORMING COMPRESSIBLE COMPACTS

R.G. Holdich \& G. Butt 
$\underline{\text { Figure } 3} \quad \underline{\text { Schematic diagram of sedimentation equipment }}$

EXPERIMENTAL AND NUMERICAL ANALYSIS OF A SEDIMENTATION FORMING COMPRESSIBLE COMPACTS

R.G. Holdich \& G. Butt 


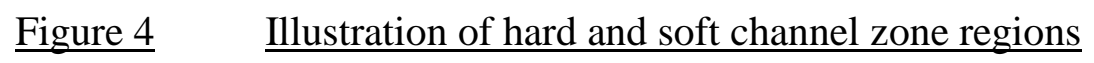

EXPERIMENTAL AND NUMERICAL ANALYSIS OF A SEDIMENTATION FORMING COMPRESSIBLE COMPACTS

R.G. Holdich \& G. Butt 
Figure 5 Measured lines of constant solids concentration and observed interface and channel heights from the base with time for a sedimentation at an initial concentration of $7.2 \% \mathrm{v} / \mathrm{v}$

EXPERIMENTAL AND NUMERICAL ANALYSIS OF A SEDIMENTATION FORMING COMPRESSIBLE COMPACTS

R.G. Holdich \& G. Butt 
Figure $6 \quad$ Variation in Kozeny coefficient for talc with solid concentration obtained from permeability data using two different experimental techniques

EXPERIMENTAL AND NUMERICAL ANALYSIS OF A SEDIMENTATION FORMING COMPRESSIBLE COMPACTS

R.G. Holdich \& G. Butt 
Figure $7 \quad$ Measured and predicted lines of constant solids concentration for a talc sedimentation at an initial concentration of $5.2 \% \mathrm{v} / \mathrm{v}$

\section{EXPERIMENTAL AND NUMERICAL ANALYSIS OF A SEDIMENTATION} FORMING COMPRESSIBLE COMPACTS

R.G. Holdich \& G. Butt 
Figure $8 \quad$ Measured and predicted talc concentration profile during a talc sedimentation at an initial concentration of $5.2 \% \mathrm{v} / \mathrm{v}$

EXPERIMENTAL AND NUMERICAL ANALYSIS OF A SEDIMENTATION FORMING COMPRESSIBLE COMPACTS

R.G. Holdich \& G. Butt 
$\underline{\text { Figure } 9} \quad \underline{\text { Measured and predicted lines of constant solids concentration for a talc }}$ sedimentation at an initial concentration of $11.2 \% \mathrm{v} / \mathrm{v}$

EXPERIMENTAL AND NUMERICAL ANALYSIS OF A SEDIMENTATION FORMING COMPRESSIBLE COMPACTS

R.G. Holdich \& G. Butt 
Figure 10 Hydrostatic pressures and interface height for a talc sedimentation at an initial concentration of $17.2 \% \mathrm{v} / \mathrm{v}$

\section{EXPERIMENTAL AND NUMERICAL ANALYSIS OF A SEDIMENTATION} FORMING COMPRESSIBLE COMPACTS

R.G. Holdich \& G. Butt 